\title{
Civilisations
}

Revue internationale d'anthropologie et de sciences

humaines

56 | 2007

Après la catastrophe

\section{Au bon vieux temps de la coopérative : à propos de la nostalgie dans un district rural de la Mongolie contemporaine}

Laurent Legrain

\section{OpenEdition}

Journals

Édition électronique

URL : http://journals.openedition.org/civilisations/146

DOI : $10.4000 /$ civilisations. 146

ISSN : 2032-0442

\section{Éditeur}

Institut de sociologie de l'Université Libre de Bruxelles

\section{Édition imprimée}

Date de publication : 1 décembre 2007

Pagination : 103-120

ISBN : 2-87263-016-3

ISSN : 0009-8140

\section{Référence électronique}

Laurent Legrain, «Au bon vieux temps de la coopérative : à propos de la nostalgie dans un district rural de la Mongolie contemporaine », Civilisations [En ligne], 56 | 2007, mis en ligne le 01 décembre 2010, consulté le 02 mai 2019. URL : http://journals.openedition.org/civilisations/146 ; DOI : 10.4000/ civilisations. 146 


\title{
Au bon vieux temps de la coopérative \\ A propos de la nostalgie dans un district rural de la Mongolie contemporaine
}

\author{
Laurent LEGRAIN
}

Résumé : A partir du récit d'un habitant d'un district rural de la Mongolie septentrionale et de vignettes ethnographiques tirées de la vie quotidienne de ces hautes steppes montagneuses, cet article vise à rendre compte de l'émergence, dans les tranches jeunes de la population, d'une tonalité nostalgique qui parasite le quotidien. Ces relectures historiques teintées de nostalgie se transmettent-elles aux générations montantes, par quels médiums, dans quelles situations, à travers quels réseaux sociaux? Comment s'articulent et se renforcent récits nostalgiques et situations quotidiennes? L'analyse proposée dans la deuxième partie de l'article tente de révéler le rôle que joue l'environnement matériel dans la manière dont les habitants du district se souviennent et donnent du sens à une histoire parfois antérieure à leur naissance.

Mot-clés : Mongolie, Darkhad, mémoire, nostalgie, transmission, politique culturelle soviétique.

Summary: From the narrative of an inhabitant of a rural district of northern Mongolia and from ethnographical vignettes drawn from the everyday life of these high mountainous steppes, this paper aims to account for the emergence, in the younger parts of the population, of a nostalgic tonality which parasitises everyday life. Do these historical re-readings, tainted with nostalgia, get transmitted to future generations? Through which media, in which situations, through which social networks? How do nostalgic accounts and everyday situations get intertwined and ultimately reinforce each other? The analysis in the second part of the paper attempts to reveal the role played by the material world in the way these people are reminded and make sense of a history at times predating their birth.

Key words: Mongolia, Darkhad, memory, nostalgia, transmission, soviet cultural policies. 
Aryn khargyn süljegeer

Avch l tavch l yavlaa

Altan Taldaa orokh 1 geed

Aminchlan zakhichgaad irlee

Zavryn khargyn süljegeer

Zavch l tavch l yavna

Zaaval negdeldee orokh l geed

Metgelee tavj l yavna uu
Je suis allé ici et là

et j'ai fait toutes sortes de choses mais aujourd'hui je suis venu les implorer de me faire membre d'Altan tal Je suis allé ici et là

et j'ai fait toutes sortes de choses je suis maintenant venu leur demander de sans faute me faire entrer dans la coopérative,

Chanson populaire darkhad

\section{Introduction}

T 'histoire moderne de la Mongolie est tourmentée et constituée de changements de Lap surprenants et souvent meurtriers (Bawden 1968). Au début du siècle passé, elle connaît deux révolutions en une décade. La première l'affranchit de la dynastie des Qing (1644-1911). Elle porte les espoirs grandioses du pan-mongolisme et de l'indépendance. Mais les Chinois seront vite de retour et en 1921, un parti révolutionnaire mongol infiniment minoritaire implore l'aide des bolcheviks victorieux en Russie pour chasser de son territoire l'armée chinoise et les restes de l'armée tsariste. La Mongolie deviendra le premier satellite de la nouvelle Union des Républiques Socialistes Soviétiques. Elle connaitra les affres du régime de terreur stalinien et les excès de la dictature du parti unique. Mais son importance géopolitique à l'époque du conflit sinosoviétique entraînera une politique de développement volontariste de la part des autorités de Moscou. L'industrie, le secteur militaire, l'élevage feront l'objet d'investissements considérables. La Mongolie, jusqu'à la transition pacifique de 1991, sera en tous domaines dépendante de l'aide financière et de la tutelle idéologique $\mathrm{du}$ « grand frère soviétique ».

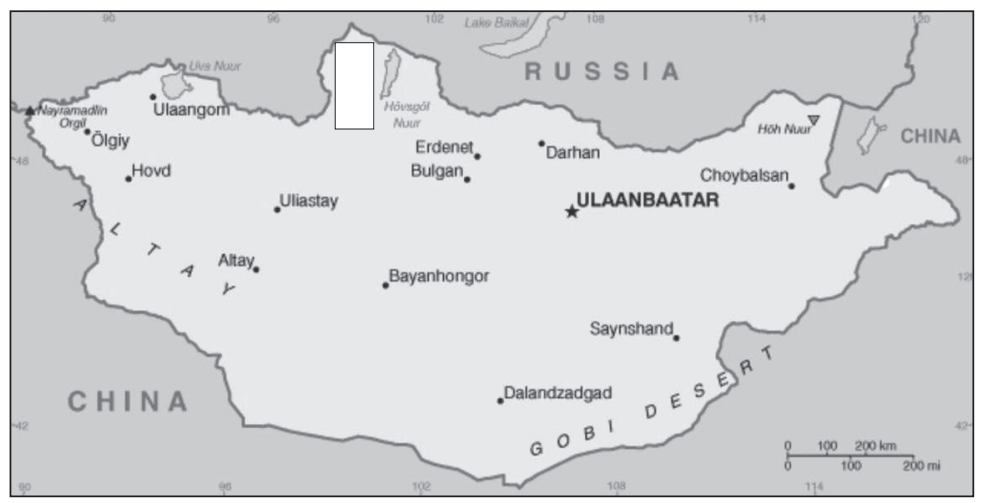

Source: https://www.cia.gov/library/publications/the-world-factbook/geos/mg.html 
Cet article tente de rendre compte de la manière par laquelle les habitants de Rinchinlkhümbe ${ }^{1}$, petit district rural du nord de la Mongolie à forte composante darkhad ${ }^{2}$, appréhendent leur passé socialiste, et particulièrement celui d'Altan-tal « Plaine dorée », la coopérative d'élevage du district créée en 1956 et démantelée en 1991. A travers le témoignage d'un homme, je voudrais mettre en avant trois nœuds narratifs qui apparaissent comme des points de passage obligés de toute mise en récit de l'histoire récente de la région. Lorsque, dans ce district, les résidents évoquent les politiques socialistes et leurs impacts locaux, les mêmes embranchements structurent leur discours. Ces nœuds narratifs une fois passés, la relecture historique aboutit souvent à jeter un regard nostalgique sur la deuxième collectivisation de la seconde moitié des années cinquante. Tant et si bien qu'en suivant mes interlocuteurs, il m'est apparu que « la catastrophe » pour reprendre la thématique fédératrice de ce numéro qualifie mieux, à l'heure actuelle, l'interminable transition démocratique que toute autre période historique. C'est cette posture émique que je reprends à mon compte ici. La catastrophe, pour la plupart des habitants de cette petite localité rurale, se vit dans la banalité quotidienne du sentiment de la chute, de l'abandon et dans le regret d'un passé encore proche. Ce qui a particulièrement attiré mon attention est le fait que ce sentiment, cette tonalité affective semblent frapper de plein fouet non point les bâtisseurs de coopératives, dont la génération s'éteint peu à peu, mais leurs enfants aujourd'hui dans la force de l'âge. Dès lors, la question posée ici est également celle de la transmission d'une mémoire historique et des bases sur lesquelles s'échafaude cette nostalgie. Dans quelles situations, par quels médiums, à travers quels réseaux sociaux émerge cette tonalité qui teinte l'expérience ordinaire de cette population.

La première partie de cet article, la plus longue, prend appui sur le récit d'un habitant du district en favorisant deux lignes de lecture : a) la mise au jour de nœuds narratifs et b) la confrontation de politiques étatiques globales, centralisées et dogmatiques avec une multitude d'arrangements et de résistances locales. En procédant de la sorte, j'espère mettre en lumière la « charpente principale d'une histoire reconstruite » (Kundera 1979 : 145). C'est, à mon sens, uniquement à partir d'un tel travail que l'on pourra appréhender la nature de cette tonalité nostalgique. Mais encore faut-il comprendre comment cette « fragile charpente » se voit dotée de murs et devient ainsi l'habitation dans laquelle les gens du district vivent leur histoire (ibid. : 147).

La deuxième partie tente, à titre encore très exploratoire, d'appréhender au niveau phénoménologique l'un des mécanismes qui donne de la consistance au récit dans l'expérience quotidienne des résidents. Ce faisant, mon travail se distingue des tentatives

1 Le système de transcription des termes mongols utilisé ici est le suivant :

$$
\begin{array}{lc}
\mathrm{Sh}=\mathrm{ch} & \ddot{\mathrm{U}}=\mathrm{ou} \\
\mathrm{Ch}=\mathrm{tch} & \mathrm{U}=\mathrm{au} \\
\mathrm{J}=\mathrm{dj} & \ddot{O}=\mathrm{eu} \\
\mathrm{Z}=\mathrm{dz} & \\
\text { Les autres lettres suivent la prononciation française. }
\end{array}
$$

2 Les Darkhad sont une population très minoritaire de Mongolie septentrionale. Ils sont largement implantés dans les trois districts à l'ouest du lac Khövsgöl où ils représentent de $70 \%$ à $80 \%$ de la population. Les particularités sociales et historiques de ce groupe seront abordées dans cet article au fur et à mesure de l'analyse du récit d'un habitant du district. 
d'explication sociologique du mal-être des zones rurales de Mongolie principalement proposées dans la littérature anglo-saxonne (Humphrey \& Sneath 1999, Bulag 1998, Potkanski \& Szynkiewicz 1993), et ceci en prenant appui à la fois sur mes expériences de terrain et sur une analyse théorique qui sera exposée plus loin. Les éléments sociologiques comme l'abandon de l'élevage spécialisé, la clôture des réseaux de sociabilité, les pénuries de biens de consommations suffisent-ils à expliquer la nostalgie du passé socialiste dans lequel est plongé le district? Ce qui semble colorer l'expérience quotidienne d'une tonalité si négative n'est pas de l'ordre d'un conflit avec un voisin ou ne résulte pas de la dégradation d'un pâturage autrefois plus riche, ni d'une difficulté à se procurer l'un ou l'autre bien de consommation. Ces événements, qui sont à la base des développements sociologiques, sont souvent considérés comme des épiphénomènes par mes interlocuteurs et ne semblent devoir figurer dans leur témoignage que pour guider la conversation vers Altan Tal, le projet coopératif, démantelé par ses membres en 1991.

\section{Le récit}

\section{Premier nœud narratif : le soldat et le badarchi}

En 1923, deux ans après la victoire des révolutionnaires, le comité central autorise les grands et les petits rassemblements festifs (tsengeldekh khüreelen). Dans ce nouveau contexte, toute fête populaire, toute assemblée doit mettre en lumière la nouvelle idéologie (Pegg $2001: 253)^{3}$. Les districts du nord de la province de Khövsgöl voient rapidement arriver leur premier « coin rouge» (Ulaan bulan) : ce sont des yourtes (ger) que l'on déplace au gré des visites rendues aux différentes populations. On y joue de courtes pièces de théâtre ou de la musique et, à l'aide de panneaux didactiques, on tente de faire comprendre les bases des changements idéologiques survenus dans le pays ${ }^{4}$.

Targan Bayraa, résident du bourg au centre du district, se souvient de ce que lui en disait son grand-père :

Les gens entraient dans la yourte ou s'alignaient à l'extérieur s'il faisait beau. C'était à cette époque un gars de l'armée qui s'occupait du matériel. Il jouait, sur base de partitions, des mélodies de Stravinsky ou de Prokofiev. Vers la fin des années vingt, le père de mon grand-père y a écouté, sur un phonogramme, les œuvres symphoniques de grands compositeurs russes. Il arrivait que ce soldat raconte sa formation, explique les paroles de Lénine et la grande révolution d'octobre.

Le bras exécutif du pouvoir, lorsqu'il arrive dans ces régions reculées, n'est à cette époque pas très musclé : un soldat, porteur de son savoir propre et ayant reçu une courte formation portant sur les piliers du dogme léniniste, les disques de grands

3 Plus précisément, ces fêtes et la politique artistique doivent faire la propagande de la ligne du parti (Namyn üzel surtalchlakh yostoi). Mais les moyens pour arriver à ces fins ne sont pas encore stabilisés. Comme le note Stefan Morawski : «Avant les années trente, une lutte ouverte entre différents courants artistiques se poursuivait à Moscou » (1961: 126).

4 Selon le très officiel ouvrage Soyolyn tü̈̈kh (Dorjnamjim 1980) qui retrace l'histoire des progrès culturels de la Mongolie socialiste, ce n'est qu'en 1926 que se généralise l'envoi de soldats chargés de diffuser les idéaux socialistes par le médium artistique. Mais deux raisons me font penser qu'un envoyé de l'Etat pouvait se trouver dans la région darkhad avant cette date. D'abord le peuple darkhad est un peuple périphérique qui était considéré comme « arriéré » et ensuite, la répression sanglante de mouvements populaires dans la république de Touva en 1924 avait transformé cette région en poudrière. 
compositeurs russes, un phonogramme à manivelle, voilà l'appareil d'Etat destiné à éduquer les populations de vallées immenses qui, depuis 1688 et jusqu'à la révolution, se définissaient comme les disciples (shav) d'un Etat ecclésiastique, le darkhad ikh shav dépendant du Khutugtu5, l'autorité lamaïque suprême de Mongolie. Ce soldat n'arrive cependant pas seul. Le nouveau régime tente à l'époque de s'attirer la sympathie du peuple notamment par la suppression des lourdes taxes levées par le clergé. Il impose une nouvelle organisation administrative : chaque groupe de dix familles (arvan) peut élire un représentant qui siègera au conseil démocratique, lequel a pour fonction de discuter des affaires de la circonscription. Des responsables choisis par le régime dans la tranche la moins éduquée de la population se retrouvent directement au sommet de la nouvelle pyramide hiérarchique. En 1926, une partie des bâtiments du monastère de Zölöön, dans le district de Rinchinlkhümbe, est transformée en école primaire (Badamkhatan 1987 : 35) destinée à accueillir vingt-cinq élèves.

Les changements dans l'organisation sociale sont donc de taille, mais les anciens rapports de pouvoir se glissent dans la nouvelle structure en rendant souvent caduques les intentions des législateurs (le Comité central du parti). Très vite, par exemple, la hiérarchie d'âge est tolérée « si elle était subsumée dans le fonctionnement de l'appareil du parti » (Pedersen 2004 : 53). Dans les assemblées locales, seuls les nobles sont élus et le gouvernement doit annuler le résultat des élections à plusieurs reprises (Bawden 1968 : 256-257). Jusqu'en 1928, date à laquelle la Mongolie passa sous complète dépendance du communisme stalinien, on trouvait en Mongolie un parti révolutionnaire comptant en son sein beaucoup de lamas et de croyants. Des intellectuels comme le Bouriate Jamtsarano, alors membre du Comité central, minimisaient les contradictions entre marxisme et bouddhisme et tentaient de mettre en avant les spécificités du développement socialiste de la Mongolie ${ }^{6}$ (ibid. : 286). Ce qui est certain, c'est que l'assaut, lancé par les révolutionnaires contre cet Etat dans l'Etat que représentait le clergé lamaïque, ne fut pas mené dans ces sept premières années de socialisme.

Rinchinlkhümbe est à l'époque la localité la plus importante de la région, et le grand monastère de Zöölön est une plaque tournante du commerce au sud du lac Baïkal. Son éloignement des limites septentrionales de l'ancien empire chinois explique sans doute

5 En 1639 le khan Gombodorji (l'un des multiples descendants de Gengis khan) qui règne sur le khanat de Tüshetü fait de son fils âgé de trois ans l'autorité suprême d'une Eglise bouddhique institutionnalisée et reconnue par les deux autres khanats khalkha (à cette époque les khanats représentaient, plus qu'une unité territoriale, l'ensemble des sujets et des avoirs d'un khan). Le territoire de nomadisation de ces trois khanats équivaut approximativement à l'actuelle Mongolie, à l'exclusion des trois provinces les plus occidentales : Khovd, Bayan-Ölgii et Uuvs. Les intentions du khan sont éminemment politiques. Il cherche à instaurer une force attractive dans le but d'unir son peuple qui, bien que payant un tribut aux Mandchous, jouit encore à l'époque d'une certaine autonomie. Il est probable que la donation au hiérarque des territoires darkhad quelques décades plus tard ait également répondu à des visées politiques. Au premier chef, il s'agissait de protéger ces vallées de l'influence de plus en plus forte des colons russes en les faisant passer sous le contrôle d'un homme qui conciliait à la fois le prestige de la descendance genkhiskhanide et l'autorité spirituelle d'un Bouddha réincarné (Bawden, $1968: 62$ ).

6 On trouve dans l'ouvrage de Bawden (1968: 281) une citation qui montre bien à quel point certains membres du gouvernement révolutionnaire cherchaient un palliatif à l'intransigeance de l'athéisme marxiste: "Seeing that the basic aims of our party and of Buddhism are both the welfare of the people, there is no conflict between the two of them. They are mutually compatible (...). It is a special case that in Russia religion is the opium of the people (...)". 
que l'on y trouvait dès la première révolution de 1911 un comptoir russe à côté des cinq comptoirs chinois ${ }^{7}$. Comme le montre le plan ci-dessous, réalisé par un moine officiant à Zöölön au début du siècle passé (Dundiv : 1912), des habitations de bois fixes se sont concentrées autour du monastère.

[photo 3]

Pourtant, ce n'est pas là que s'installe notre soldat en charge de distiller la culture et l'idéologie du parti dans ces hautes steppes :

Il arrivait puis repartait sans qu'on entende plus parler de lui pendant quelques semaines. Les gens disaient à cette époque qu 'il repartait à Mörön ou Khatgal pour y recevoir de nouvelles instructions.

C'est en effet à Khatgal au sud du lac Khövsgöl que les communistes ont installé une garnison (khoroo) en 1921, après leur victoire sur les restes de l'armée blanche du baron Ungern von Sternberg (Atlas de la province de Khövsgöl 1988). Pourquoi n'avaient-ils pas cantonné leurs troupes à Rinchinlkhümbe, plus à même d'accueillir une concentration massive d'hommes ? La réponse ne peut être que spéculative, mais on peut supposer qu'ils n'auraient pas été les bienvenus. Le gouvernement maintenait une certaine pression sur l'Eglise ${ }^{8}$ et, ce faisant, il se heurtait à l'opposition des badarchi. Ces moines pauvres et ambulants passant de foyer en foyer pour dire l'avenir, guérir et pratiquer de menus rituels apotropaïques faisaient une contre-propagande intensive en présentant les révolutionnaires comme des ignorants illettrés et athéistes ${ }^{9}$. Dans leur travail de sape de la nouvelle idéologie, ces derniers pouvaient s'appuyer sur des contes et des légendes (souvent d'origine indienne et tibétaine) qui font partie du bagage culturel de chacun (Bawden 1968 : 265, Legrand 1981). De plus, étant donné la ligne stratégique du parti, qui consistait à choisir les dirigeants des cellules locales dans la tranche la moins éduquée de la population, les assertions des badarchi sur l'ignorance des cadres de la nouvelle politique se trouvaient confirmées d'elles-mêmes.

\section{Une narration fantôme : l'oubli inoubliable (Kundera 2003)}

Le deuxième nœud narratif possède un statut particulier. Il garde cette appellation du fait de son influence structurante sur tout discours historique énoncé dans ces hautes vallées septentrionales, mais la narration y brille par son absence. En effet, dans le cas qui nous occupe, de par la capacité du récit de jouer sur les cadres temporels de toute

7 Sous la dynastie des Qing, les maisons commerciales chinoises se sont progressivement infiltrées en Mongolie. Leur objectif était de monopoliser le commerce d'une bannière (principale organisation territoriale et militaire) jusqu'à devenir le créancier du prince en place. Selon Charles Bawden (1968 : 96) l'administration impériale tenta à plusieurs reprises de réglementer ces pratiques, mais sans grand succès. Voir aussi à ce propos Wheeler $(2003: 220)$.

8 Les modes de pression étaient divers : des taxes, la réduction drastique des taux d'intérêts pratiqués par les monastères dans leur activités usurières, l'exemption des devoirs incombant aux petits éleveurs (tels que la garde des troupeaux appartenant aux lamas), des écrits et des pièces de théâtre satiriques.

9 Des anecdotes de terrain semblent confirmer l'intense activité des badarchi. Aujourd'hui la région étant devenue touristique, il n'est pas rare que les habitants, à la vue d'un touriste isolé marchant dans la steppe en portant son sac à dos, le surnomment « badarchi » dans de grands éclats de rire. 
expérience, les paroles de mon interlocuteur vont, d'un seul silence entre deux phrases, nous faire faire un bond en avant de vingt-huit ans. A peine a-t-il fini d'évoquer le soldat $\mathrm{du}$ « coin rouge », ses partitions et ses disques, que Targan Bayraa poursuit en disant : «Après lui, nos pères et nos aînés fondèrent Altan-tal en 1956, puis le centre culturel en 1965 ». Il passe ainsi sous silence les périodes les plus tumultueuses de l'histoire de la Mongolie révolutionnaire. L'objectif n'étant pas dans cet article de faire un survol des différentes étapes historiques de la Mongolie moderne, je me contenterai ici de mettre en regard cet épisode du récit (ou du non-récit : un oubli de presque trente ans d'histoire) et la véritable politique de tabula rasa opérée avec un certain succès dans les années trente et quarante par le gouvernement socialiste.

L'ethnologue mongol Badamkhatan (1987 :36) emploie lui aussi une formule laconique pour évoquer la fin des activités religieuses dans le district : " Le célèbre monastère de Zöölön fut fermé en 1938 : ses lamas entrèrent dans le collectif de menuiserie, textile et alimentation créé sur place (à sa fermeture, le monastère abritait mille cinq cents lamas) ». Il est difficile de collecter des informations sur la fermeture des monastères. A l'échelon national, il n'existe à ma connaissance aucun document. Batchuulun est un ancien membre de la coopérative Altan-tal, aujourd'hui installé pour ses vieux jours à Oulan-Bator. Fervent nostalgique de la période coopérative, il incorpore pourtant dans ses écrits (2004) de longues listes de noms de lamas exécutés à cette époque. La tradition orale va dans ce sens également. Les gens parlent de nombreuses exécutions sommaires devant le monastère, de traques et d'arrestations, de déportations, en bref d'une période de terreur :

Des membres des cellules du parti, venus des provinces avoisinantes pour ne pas qu'on puisse les reconnaître, se donnaient l'apparence de badarchi et visitaient nos campements. Dans la soirée, ils évoquaient la religion. Si un membre du campement posait des questions, portait trop d'attention aux dires de l'espion, il était arrêté quelques jours plus tard et jeté en prison » témoigne une femme des environs du district Tsagaan-nuur (le lac Blanc). "Les enfants qui jouaient autour de l'ancien site du monastère trouvaient des statues de Bouddha décapitées et des objets de culte brisés ».

Le règne de Choïbalsan, l'épigone mongol de Staline, qui prend au début des années trente les rênes du pouvoir et qui ne les lâche qu'à sa mort en 1952, correspond à l'épisode de la destruction totale du vieil ordre social : clergé et aristocratie (Bawden 1968, Batbayar 1999).

Sharlai, un homme d'une septantaine d'année se souvient de l'attitude des lamas lorsque, après avoir purgé des peines de dix à vingt ans de prison, ils revenaient dans leur famille :

Pas loin de mon campement d'été, il y avait une famille dont un des fils avait été officiant au monastère. Lorsqu'il est revenu, après avoir purgé sa peine, son père était mort depuis cinq ans. J'allais souvent leur prêter main forte pour les travaux du printemps. Jamais il n'a reparlé du monastère, ni à moi, ni à ses frères et sœurs, ni à personne et aujourd'hui la grandeur des lieux s'est perdue dans l'oubli parce qu'aucun des survivants ne voulait en reparler, tu comprends?

Pour les jeunes générations, il n’y a aucune narration disponible de ces années noires. Cette tranche d'histoire non formulée plutôt qu'oubliée ouvre le champ des interprétations vers deux possibilités. La première interprétation nous ramène à un schéma historique qui 
hante toute la littérature des années socialistes. En effet, bon nombre de mes interlocuteurs combattent parfois énergiquement la lecture historique qui fait des purges des années 1930 la concrétisation meurtrière d'intentions bien plus anciennes dans le programme du parti révolutionnaire et constitutives de l'idéologie soviétique. Pour beaucoup d'adultes et de jeunes du district, les purges sont une «erreur de jeunesse » d'un parti dont la gouvernance n'a cessé de s'améliorer de la révolution de 1921 au démantèlement du bloc soviétique en 1989-1990. On reconnaît en filigrane cette idée du "progrès constant » propre aux lectures idéologiques de l'histoire. D'autre part, et nous entrons là dans la deuxième ligne d'interprétation, cet angle mort dans l'appréhension de l'histoire locale permet de relier les questions relatives au monastère à des questions identitaires tout actuelles. Pour la plupart des gens du district, la principale question à résoudre est la suivante : « Pourquoi avoir rasé le monastère darkhad jusqu'à la dernière pierre alors qu'en d'autres lieux, en Arkhangai (la province méridionale limitrophe) par exemple, l'armée et la police avaient laissé des bâtiments debout »? La réponse est toujours la même : non seulement Zöölön était un monastère puissant, mais il était également le principal lieu de culte d'une minorité nationale (yastan), les Darkhad ${ }^{10}$, parvenue à son apogée culturelle et jalousée par le pouvoir en place. Ces années sont en effet celles où s'est poursuivi sur une large échelle le processus dénommé « khalkhisation » par l'anthropologue Uradyn Bulag (Bulag 1998 : 34). Le principe en est simple : à chaque pays correspond un peuple (ündesten ${ }^{11}$ ) et une langue. En Mongolie, c'est la langue comme la culture matérielle et artistique des Khalkha (le groupe ethnique largement dominant occupant les territoires centraux) qui deviennent les modèles à reproduire et à incorporer. Les minorités (qui forment une mosaïque impressionnante sur le territoire mongol) doivent être assimilées et disparaître. La formule « nationale dans sa forme, socialiste dans son contenu » devient le leitmotiv des politiques culturelles. Mais comme nous le verrons dans la section suivante, même après les années de terreur du règne de Choïbalsan, l'Etat ne parviendra pas à éradiquer les représentations par lesquelles les Darkhad se pensent comme une minorité nationale particulièrement remarquable par sa « qualité » et sa spiritualité. Traits qui, dans leur lecture de l'histoire, expliquent pourquoi, tant dans le régime monastique que dans le système soviétique ils parvinrent à se démarquer de leurs voisins par des réalisations enviables. Ce silence qui constitue paradoxalement l'un des nœuds narratifs de leur discours, leur permet de penser cette période historique trouble en recourant à une opposition qui est, elle, très claire : les Darkhad et leur nature spécifique versus les autres populations de Mongolie. Alors qu'une version plus documentée de la même séquence historique $^{12}$ pourrait sans disqualifier cette opposition en mobiliser d'autres, comme par exemple le socialisme versus les figures de l'ordre social antérieur, le socialisme versus la religiosité.

Zöölön est plus souvent appelé darkhadyn khüree : le monastère darkhad.

12 Il apparaît notamment que de nombreux Darkhad ont pris part à la destruction de Zöölön. En 1938, l'alternative à l'ordre social monastique que représentait l'idéologie socialiste avait semé le trouble chez bon nombre de Darkhad. Il est fort probable qu'à l'époque l'opposition Darkhad - non-Darkhad n'ait joué dans cette affaire qu'un rôle mineur. 


\section{A propos du centre culturel d'Altan-tal : l'âge d'or du territoire darkhad}

Arrivé à ce troisième nœud narratif, le récit de Targan Bayraa s'emballe. J'en transcris ci-dessous l'intégralité en conservant le foisonnement des détails. La période socialiste est trop souvent regardée comme un bloc monolithique de politiques centralisées, coordonnées et infaillibles, alors que l'extrait qui suit montre à quel point les aménagements locaux sont importants.

\section{« Les centres culturels étaient les veines et le cœur des politiques socialistes »}

Le 7 novembre 1965, le centre culturel d'Altan-tal a ouvert ses portes en présence de tous les membres de la coopérative. Yondon, alors dirigeant de l'organisation, prit le camion de la quatrième brigade et rapporta d'Oulan-Bator les énormes lustres qui pendent encore dans la salle des concerts. Cette année-là, tous ceux qui avaient participé à la construction du centre furent récompensés par le don de feutre et de chaussures, qui valaient à l'époque quarante-cinq tögrög. La personne qui dirigeait le travail, un certain Shargabumdzad de la vallée de Khogrog reçut un salaire de mille tögrög, qui représentait à peu près deux mois de traitement pour une personne bien diplômée. Le centre culturel est un énorme bâtiment. Depuis cette époque jusqu'à l'heure actuelle, il n'a jamais dû être réparé. C'est étrange n'est-ce pas, de trouver dans un petit district comme le nôtre un centre culturel de cette taille? Mais Yondon était un grand homme. Lorsqu'il dirigea la province de Zavkhan dans les années cinquante, il prit une photo du palais de la culture d'Uliastai, le chef lieu de la province. On construisit le centre culturel sur la base de la photo prise à Zavkhan. Il arrivait que certains membres de la coopérative critiquent Yondon en lui disant: "Pourquoi construire ici un tel bâtiment? Les habitants de ces vallées sont dispersés...avec qui allons nous le remplir?" Quelques décades se sont écoulées et les gens ont maintenant compris qu'il avait raison. Le centre culturel a quarante ans et il est toujours sur pied. Il est bourré à craquer lorsqu'une activité y est organisée.

Des tracteurs sont partis abattre de grands arbres que l'on trouve le long du lac - ce sont les arbres les plus hauts de Mongolie - pour les ficher dans la terre juste à l'entrée du centre culturel. Ils y sont encore! En un an, notre centre culturel était construit. Toutes les familles que tu peux rencontrer ici ont contribué d'une manière ou d'une autre à cet effort.

Au moment où s'organise Altan-tal au début de l'année 1956, Yondon, selon ses propres écrits, est à l'origine du rassemblement des premiers éleveurs (Yondon 2004). Il restera un homme fort de la politique locale jusqu'à sa mort en 2002. Or, la stratégie de ce deuxième mouvement de collectivisation en Mongolie, mouvement à nouveau décidé unilatéralement par le Comité central du parti ${ }^{13}$, consiste, du moins en théorie, à ne jamais placer des gens du cru à la tête des coopératives, et ceci pour éviter toutes velléités de localisme (nutgarakh üzel) (Bulag 1998 : 43). Les dimensions et la décoration intérieure des centres culturels ne sont pas livrées à la fantaisie ou au rêve grandiloquent d'individualités visionnaires. Pourtant, Yondon semble avoir les mains libres lorsqu'il indique sur photo le style du bâtiment qu'il veut réaliser, part avec le camion de la brigade

13 Le souci du Comité central est purement dogmatique. Il veut suivre de près le sentier tracé par la collectivisation des zones rurales de l'Union soviétique. 
chercher des lustres qui peuvent rivaliser avec ceux du grand théâtre d'Oulan-Bator, ou ordonne de déraciner les plus grands arbres de Mongolie et paye une coquette somme à son maître d'œuvre. L'extrait donne l'impression qu'en tant que dirigeant de la coopérative, il est l'unique maître à bord. S'agit-il d'un cas particulier ? Devons-nous arguer, comme le font les habitants du district, de l'amitié qui le lie à Tsedenbal (futur Premier ministre et homme fort du pays jusqu'à la fin du communisme) ? C'est un homme influent (il fut le chef d'une province entière) mais ce n'est pas la seule explication possible. Je pense qu'il existe une autre manière d'interpréter ces événements. J'en livre ici une grille de lecture encore incomplète. Contrôler les coopératives demandait un appareil administratif considérable que l'Etat n'était pas toujours capable de mettre en place. Lorsque ces organisations locales réalisaient les chiffres du plan quinquennal, comme ce fut le cas pour Altan-tal, il est probable que les contrôles administratifs se soient assouplis. De manière générale, la pléthore de procédures et d'autorisations nécessaires à toute entreprise aussi banale fût-elle contraignait les individus à « s'arranger », et ceci à tous les niveaux de pouvoirs (Humphrey \& Sneath 1999 : 142). De plus, l'image d'une coopérative pimpante, réalisant haut la main les chiffres du plan était utilisée pour blâmer celles qui ne parvenaient pas à de tels résultats. C'est ainsi que la coopérative Jargalant Am'dral (Vie heureuse) du district d'Ulaan-uul « Montagne rouge » souffrit par deux fois de la comparaison avec Plaine dorée et connut deux changements successifs de dirigeants. La concurrence jouait du reste à tous les échelons de l'organisation : les provinces entre elles, les coopératives entre elles, les travailleurs entre eux. L'octroi de médailles et de titres rendu public par la presse nationale ${ }^{14}$ placardée dans tous les centres culturels ${ }^{15}$ maintenait constamment présente à l'esprit de chacun la compétitivité dont dépendait la bonne marche du système.

Retenons également pour la suite des développements, l'insistance de Targan Bayraa à attirer mon attention sur la solidité du bâtiment et son utilité actuelle.

\section{« Le plus important est de faire vivre la culture »}

Yondon n'en est pas resté là. Il portait un grand intérêt à l'art et à la politique. Il s'est mis en devoir de récolter des instruments de musique que même le Palais de la culture (Soyolyn ordon) de Mörön ne possédait pas. Tu dois te rendre compte qu'à partir de ce moment-là, les artistes darkhad ont commencé à tourner dans toutes les provinces mongoles et même à l'étranger. Une femme du nom de Zunduganlanz fut la première à chanter des chants darkhad devant des jeunes de tous les pays du bloc socialiste dans un festival nommé "Le grand Festival de la jeunesse mondiale " (Delkhiig zaluuchuud oyutnii ikh naadam). Elle s'y vit décerner la médaille de bronze. Tserempei, un chanteur, remporta la médaille d'argent au « Grand festival de la jeunesse mongole » (Mongolyn zaluuchuud ikh naadam) en 1968. Grâce à eux, la coopérative obtint un camion et un tracteur. Les vallées darkhad sont le berceau

L'annonce incluait une photo du visage de l'heureux élu. Lors des fêtes de fin d'année, ces travailleurs méritants rejoignaient le Palais de la culture de Mörön pour y être acclamés et recevoir leur titre des mains d'un officiel important. Aujourd'hui le souvenir des petites tactiques mises au point pour truquer les résultats de sa production personnelle et ainsi accéder au titre provoque l'hilarité générale. On a cependant pris soin au préalable de demander à l'anthropologue d'éteindre son magnétophone. cérémonie matinale portait un nom en mongol : le sonin unshlaga, « le temps de la lecture du journal ». 
de bien des talents. Depuis les années soixante et jusqu'à la fin du socialisme, mon district était fameux pour les hommes de culture (urlagyn khün) qui en étaient issus. D'autres chanteurs et musiciens sont partis pour travailler dans l'" ensemble de chants et de danses nationales » (ulsyn duu büjgiin chuulga).

Innombrables sont les médiations par lesquelles l'idée de progrès culturel est rendue présente dans le quotidien des éleveurs les plus éloignés des centres - on commémore sur un rythme effréné la naissance de chaque institution : les cinq ans de l'école, les deux ans du jardin d'enfants, les dix ans de la coopérative, de la révolution nationale ou de la révolution d'octobre. Cette notion de progrès est ainsi ce qui enrôle l'ensemble des habitants dans la politique culturelle de l'Etat et, au-delà, d'une structure englobante et fraternelle (pour peu que l'on aille dans le sens de la marche en reconnaissant en tout domaine la supériorité du grand frère soviétique). Même dans ces vallées éloignées, le nombre s'accroît de ceux qui ont vu de leurs yeux la toute proche Union des Républiques Socialistes Soviétiques ou les lointaines républiques-sœurs d'Europe de l'Est pour y étudier, y voyager ou y présenter leur folklore régional. Ainsi Baasanjav, artiste darkhad revenu de Berlin note dans son journal : « dans ce pays il existe des forêts sans montagnes et les maisons ont des couleurs plus nombreuses que celles de nos arbres ». Loin d'être intime, ce journal circule, on en parle, on le lit, on s'étonne, on s'enthousiasme, on le relit et on en débat avidement. Les fonctionnaires culturels encouragent et popularisent cette pratique à mi-chemin entre l'aide-mémoire et le journal personnel où le travailleur consigne ses impressions, ses progrès et ceux de sa coopérative. De même, les albums photos que commencent à réaliser les cadres administratifs de la coopérative fonctionnent comme autant de panoptiques qui témoignent de l'existence d'un lien national tissé lors des réunions des cellules locales du parti, des remises des décorations aux éleveurs méritants, ou des voyages officiels à Oulan-Bator. L'éleveur comme le résident du district sent par l'intermédiaire de ces dispositifs qu'il constitue l'un des maillons d'un projet nouveau. Les décrets ministériels rythment la vie saisonnière, donnent le départ des nomadisations et les soutiennent activement : des puits sont creusés sur les parcours vers les hivernages, des étapes sont instituées, qui comprennent des installations destinées aux hommes (maison de bois confortablement équipées) et aux bêtes (enclos protecteurs). Les deux chauffeurs de la coopérative, encore réputés aujourd'hui pour leur habileté à franchir les passes de montagnes devenues infranchissables à l'heure actuelle par le manque d'entretien des pistes, transportent le gros du matériel sur des tracteurs et facilitent ainsi la tâche aux éleveurs. La vie n'est pas idyllique, loin s'en faut et nombreuses sont les retenues sur salaire dues aux écarts et aux désobéissances par rapport à des ordres parfois aberrants pris à des centaines de kilomètres du terrain. Tout le monde ici se souvient par exemple de nomadisations entamées dans le blizzard, parce que l'arrêté du ministère prévoyait des pénalités salariales pour les jours de retard. Mais même dans ses aspects disciplinaires les plus durs, l'idée de l'Etat ne s'en trouve que confirmée. L'idéologie soviétique ne flotte pas au-dessus de la vie quotidienne, elle est profondément ancrée dans les actes quotidiens de tout un chacun et force l'individu à toujours évaluer ces actions et pensées par rapport à elle (Humphrey $1983: 8$ ).

\section{Analyse}

J'ai décrit jusqu'ici la configuration d'un récit. Son organisation interne, principalement fondée sur trois nœuds narratifs, qui permettent d'appréhender plus finement la « charpente 
principale d'une histoire reconstruite » (Kundera 1979 : 145). Mes interlocuteurs décrivent d'abord une sorte de tâtonnement des politiques culturelles, oublient (la plupart du temps) le traumatisme du passage sous la tutelle stalinienne et dépeignent avec un foisonnement de détails la deuxième collectivisation des années cinquante ${ }^{16}$. Ce récit en trois temps peut être qualifié de canonique, tant les nœuds qui l'organisent flèchent le parcours de la narration d'une mémoire historique largement distribuée. On en retrouve les nouaisons chez les personnes d'un âge respectable qui ont vécu au moins deux des trois périodes évoquées, mais aussi chez celles qui atteignent aujourd'hui la vingtaine ou la trentaine, voire chez les plus jeunes. Ces derniers ne peuvent cependant avoir des souvenirs directs que de la période de la perestroïka mongole (örchlön baiguulal) et de la transition. Or, les plus mélancoliques qu'il m'ait été donné de rencontrer appartiennent à ces tranches d'âge. L'objectif n'est pas ici de comprendre l'incongruité qui consiste pour ces jeunes gens à éprouver de la nostalgie pour des événements qu'ils n'ont pas vécu. Maurice Bloch, dans ce domaine, semble avoir fait la preuve qu'il n'y a dans ce fait aucune incongruité : « le souvenir né des récits peut prendre la forme de souvenirs autobiographiques » (1995: 73). Ce qui est frappant dans le cas présent, c'est que les aînés (akhmad) ne se livrent pas dans leur évocation historique à une critique cinglante du mode de vie contemporain, ni d'ailleurs à une surestimation des difficultés actuelles par rapport à celles qu'ils ont connues. Cette nostalgie obsédante semble en première analyse ne pas être transmise, mais émerger dans les représentations et les pratiques de communication qui structurent la «mémoire collective » (Halbwachs 1976) des tranches les plus jeunes. Cependant, cette notion de mémoire collective fonctionne facilement à la manière d'une boîte noire ou d'un concept peu clair (Berliner 2005) dans les analyses anthropologiques. Je voudrais, pour décortiquer le processus d'émergence de cette tonalité émotionnelle, m'en tenir au niveau phénoménologique en montrant comment, à partir de différents éléments (le récit, un contexte d'énonciation, des éléments topographiques, une configuration interactionnelle) ce sentiment se construit et se renforce en situation. Je vais donc dans la section qui suit, analyser à partir d'une situation d'énonciation comment un ensemble d'artefacts (l'agencement des bâtiments du village) devient une « prise » (Bessy et Chateauraynaud 1995) qui ancre le récit et le greffe sur des expériences perceptuelles, ou pour reprendre les termes de Milan Kundera, qui donnent des murs à la trop fragile charpente historique.

\section{En arrivant au centre...}

Autour du centre du district, formé d'une centaine de baraquements de bois, s'étendent les steppes de pâturages striées par les trajets de nomadisations d'une petite moitié des familles de Rinchinlkhümbe. Il n'est pas rare que les chefs de familles de ces différents campements (ail) rejoignent le centre pour différentes raisons, à cheval, en jeep ou à moto. Ces voyages sont rarement solitaires. Lorsqu'un aîné part vers le centre, il est toujours accompagné de jeunes qui, d'une manière ou d'une autre, font partie de son réseau d'obligations. Les trajets sont épuisants, même si les distances parcourues sont rarement supérieures à une cinquantaine de kilomètres, mais l'arrivée en vue du bourg

16 On pourrait ajouter un quatrième nœud, lui aussi présent sans pourtant être énoncé, qui concerne les années septante et quatre-vingt, toujours perçues comme le ronronnement de la coopérative et le développement croissant de la région. 
au milieu duquel trône le centre culturel est très souvent ponctuée d'une réflexion, d'une exclamation exprimant le bonheur d'être au bout du chemin : "Nous atteignons la bonne Altan-tal !» (Saikhan Altan-tal khureed irlee). Même dans les voyages les plus silencieux, cette exclamation est suivie de conversations joyeuses, de rires, de gestes amicaux, de plaisanteries, en bref d'un changement radical de l'intensité et de l'affectivité des interactions. Joie qui se prolonge souvent jusqu'à l'arrivée ${ }^{17}$.

Le contenu informatif est ici nettement moindre et beaucoup plus ambigu que ne l'est celui du récit complet ${ }^{18}$. Mais le changement de comportement interactionnel suscité par l'évocation et la vue des anciennes installations de la coopérative est, quant à lui, beaucoup plus saillant. La topographie du village, sa silhouette que coiffent les toits du centre culturel, du bâtiment de l'administration et du jardin d'enfants (tous héritages de la période soviétique) provoque l'extériorisation d'un sentiment de bien-être, aussitôt socialisé par le comportement de chacun. On trouve le même genre d'attitude dans les jeeps qui arrivent de Mörön chargées de résidents du centre partis faire des affaires au marché du chef-lieu de la province.

\section{Le pouvoir des prises}

Voilà donc un groupe d'individus, arrivant après quelques heures de route en vue d'un village et exprimant sa satisfaction à l'idée d'un repos tout proche, d'un bol de thé ou d'une rencontre attendue. La silhouette massive du centre culturel et des bâtiments attenants ne semble jouer dans cette scène qu'un rôle minime. Elle déclenche la socialisation d'un sentiment de bien-être éprouvé depuis un bon moment. Chacun connaît la route par cœur et n'a donc nul besoin de voir le village pour savoir qu' " il arrive ». C'est l'acte de parole de l'aîné qui semble lier le passé socialiste à ce sentiment de bien-être. Mais est-ce si sûr ? L'hypothèse que je voudrais avancer ici, à titre exploratoire, c'est que les artefacts hérités du passé ne sont pas les agents passifs (ou les outils) de pratiques de communication qui construisent la mémoire collective, mais qu'ils en sont également les actants ${ }^{19}{ }^{20}$. La médiation langagière n'est pas le seul élément qui invoque dans la scène le passé socialiste. Le centre culturel, le dôme du jardin d'enfant, la belle terrasse du bâtiment de l'administration, toute la topographie du village ramène également notre groupe au troisième nœud d'un récit tant de fois entendu. Dans l'exemple ci-dessus, c'est donc aussi pour partie l'agencement matériel du village qui fait le lien entre un ensemble de repères historiques conventionnels et des sensations et perceptions éprouvées dans le vif de l'action, soit la charpente et les murs pour reprendre l'image de Kundera. Le récit s'en

17 Du moins continue-t-on à se conduire de la sorte jusqu'à l'entrée au village qui marque le retour à une expansivité mieux contrôlée.

18 Il arrive souvent que l'arrivée en vue du village soit aussi le déclencheur de l'énonciation d'une partie du récit ou à tout le moins d'une anecdote de la période socialiste.

19 La notion d'《actant» est empruntée à la sémiologie de Greimas par les sociologies de la médiation (Hennion 2000, Latour 1994). Elle permet de mieux distribuer l'action entre les différents intervenants qui interagissent, en ce compris les artefacts. Chaque médiation devient actant parce qu'elle modifie l'action en cours et ne se contente pas de la transmettre telle quelle.

20 On peut trouver les germes de cette hypothèse chez Radley (1990), par exemple. Cependant, pour lui, les artefacts sont toujours les dépositaires des intentions humaines. 
trouve comme « phénoménologiquement validé ». La satisfaction ressentie, tout actuelle, est renvoyée dans le passé par les médiations de l'acte de parole et l'agencement des artefacts.

Ce rôle d'actant des artefacts m'a semblé être confirmé dans une scène étonnante dont je fus le témoin un soir de printemps. Pürev est âgée d'une trentaine d'années. Elle est une excellente chanteuse du quatrième sous-district de Rinchinlkhümbe. Un petit groupe de proches est réuni dans sa yourte. Depuis trois bonnes heures, les bols de vodka tournent et le répertoire darkhad est chanté à gorges déployées par une assemblée de plus en plus grisée par l'alcool. Entre deux chansons, alors que les conversations parallèles battent leur plein, Pürev saisit d'un mouvement brusque la médaille gagnée en Hongrie par sa mère, Zunduganlanz. Elle entame une ode joyeuse et enthousiaste, glorifiant la femme qu'était sa mère. Son discours prend rapidement comme sujet cette période de bonheur où les artistes des vallées darkhad voyageaient à l'étranger faire découvrir aux gens du vaste monde ${ }^{21}$ la beauté des chants de son peuple. Pürev finira en pleurs après quelques minutes, rageant et pestant contre cette époque où les artistes de ces vallées, pourtant aussi capables que leurs aînés, devront se satisfaire jusqu'à la fin de leurs jours du monde artistique étriqué de leurs clubs locaux respectifs.

On peut évidemment mettre cette embardée émotionnelle sur le compte de l'alcool. Mais dans la continuité de l'hypothèse du « pouvoir des prises » que j'ai tenue jusqu'ici, je convoquerai un autre type d'explication (qui s'accommode d'ailleurs très bien de la causalité d'un éthylisme avancé, mais qui la relativise significativement). L'objet saisi par Pürev ne se laisse pas seulement manipuler par l'action volitive de celle-ci. Sa volonté de louer (magtakh) sa mère se heurte à cette médaille, artefact chargé d'un sens plus large. Et c'est finalement en tant que représentant l'organisation soviétique du monde artistique que la vue de cette médaille pousse Pürev à infléchir son discours. Ce n'est plus Pürev qui saisit la médaille, mais la médaille qui saisit Pürev. Ce changement d'un sujet en complément d'objet direct n'est pas anodin. Il implique le retour « d'une certaine dose de fétichisme » dans l'analyse anthropologique (Latour 1994 : 597). Les objets «font quelque chose $»^{22}$ aux gens qui s'en saisissent.

Or, à Rinchinlkhümbe comme dans la plupart des zones rurales de Mongolie, on est entouré de ces objets légués par la période soviétique : les jeeps qui mènent les gens jusque Mörön sont les vieux modèles russe « 69 », le tracteur gagné lors du concours de chant est encore périodiquement chargé de l'herbe des fenaisons, les surplus de l'armée russe sont les meilleures affaires que l'on puisse faire sur le marché du vêtement, le maître achat, après avoir engrangé des revenus tirés de la vente du cachemire, est une moto d'un modèle intensément diffusé à l'époque soviétique. Tous ces objets sont des prises potentielles en ce qu'ils sont capables d'ancrer le troisième nœud narratif du récit dans la réalité de l'expérience quotidienne, avec sa dimension perceptuelle et émotionnelle. En devenant des prises, des supports de l'expérience, ils orientent ou font

21 J'entends ici le monde formé par les pays du bloc socialiste (Zöövlölt kholboo uls).

22 J'adapte ici à un autre objet de recherche une hypothèse de travail soutenue par le courant pragmatiste particulièrement actif en sociologie de l'art (Hennion 1993, 2000, DeNora 2002). Cependant il me semble que le troisième nœud du récit, en ce qu'il mobilise dans sa structuration une telle prolifération d'artefacts, donne à cet emprunt une pertinence analytique dont il serait dommage de ne pas tester la capacité heuristique. 
tendre les pratiques communicationnelles vers un point de fuite toujours situé dans le passé socialiste. L'hypothèse du " pouvoir des prises » déplace le centre de gravité de la recherche de l'explication sociologique du mal-être des zones rurales de Mongolie vers une tentative d'appréhension, à un niveau phénoménologique, de la construction d'une tonalité nostalgique qui parasite le quotidien.

\section{«Mieux vaut se casser le dos que de perdre ses illusions »}

A Rinchinlkhümbe, personne ne parle de catastrophe pour qualifier la transition démocratique et le passage à l'économie de marché. Interrogés de manière frontale sur les effets de cette transition, la plupart des gens parleront de «liberté des pratiques » et de « liberté d'expression » gagnées sur le totalitarisme depuis une décade seulement. Cependant, ce qui frappe dans le travail de compilation des attitudes et des témoignages observés chez les jeunes et les adultes, c'est la référence constante et nostalgique à un âge d'or situé dans l'histoire socialiste, et ceci alors même que la génération des bâtisseurs de coopératives adoptent pour leur part une attitude beaucoup plus réservée dans leur relecture historique. La critique acerbe des conditions de vie actuelle, les cris de rage et les larmes ne sont, à ma connaissance, jamais le fait des aînés de la génération d'Altantal. Mais d'où vient dès lors ce regard passéiste et émotionnellement chargé s'il n'est pas transmis ? Ou peut-être se transmet-il d'une manière moins visible que le simple codage/ décodage d'informations pertinentes ? C'est en effet cette dernière piste qui s'impose, me semble-t-il.

La manière dont les jeunes adultes se souviennent et oublient leur passé dépend entièrement des actes de paroles et des réalisations matérielles de leurs aînés, sans toutefois que cet ensemble de médiations mouvantes ne construise chez ces deux groupes la même grille d'appréhension de l'histoire. Pour comprendre comment se met en place cette dynamique de transmission/innovation, c'est autant vers le récit que vers les microévénements qu'il faut se tourner.

Dans ces hautes vallées, l'évocation du passé socialiste est presque quotidienne et toujours le troisième nœud narratif, celui qui retrace l'âge d'or de la coopérative, provoque le même emballement, le même foisonnement de détails, le même engagement méticuleux dans la mise en évidence de tout le dispositif matériel encore sur pied aujourd'hui. Mais cette charpente historique, transmise par une parole informative, serait à mon sens bien trop fragile si elle n'était vécue ou rejouée presque quotidiennement dans la vie des habitants du district. Mais quels sont les attributs qui permettent à des microévénements de rejouer une histoire passée ? Revenons sur la deuxième partie du texte et les situations qu'elle présente. Lorsqu'en arrivant au village un aîné s'exclame : « Nous atteignons la bonne Altan-tal !» Le contenu informatif de son acte de parole est dérisoire. En revanche, cette parole fait! Que fait-elle? Elle rend présent dans l'expérience actuelle non seulement la coopérative, mais aussi son caractère « bon » et donc capable d'apporter le bien-être et renvoie le réconfort ressenti en situation dans le passé socialiste. Cette performativité des actes de langage se lit, comme je l'ai précisé plus haut, dans le changement des dynamiques interactionnelles qui le suivent: dans la steppe venteuse, des hommes jusqu'ici silencieux, se mettent à plaisanter, rire de l'un ou de l'autre et se chamailler. Dans la deuxième situation évoquée, la yourte surchauffée est le théâtre du basculement d'une communauté de la joie aux pleurs et des chants à la compassion silencieuse pour l'une des leurs, offensée par le sort. 
Mais ces actes de paroles ne sont qu'un des actants parmi d'autres d'une situation complexe qui inclut bien d'autres éléments. J'ai tenté dans cet article de révéler la dynamique qui s'instaure entre au moins trois de ces actants. A côté des actes de paroles et d'une charpente historique plus ou moins sédimentée en chacun par la répétition des nœuds structurant le récit, l'hypothèse du " pouvoir des prises » laisse une place à l'influence incontrôlée de la matérialité des objets sur les manières d'envisager le passé socialiste de ces lieux.

La question de la transmission est-elle donc finalement bien posée ? A mon sens, elle ne l'est pas si l'on cherche à travers elle à séparer ce qui apparaîtrait comme la substance première et stable d'une innovation. Il serait, par exemple, faux de dire que les aînés transmettent un récit (qui reste donc égal à lui-même) et que les jeunes lui donnent une nouvelle tonalité émotionnelle. Si dans cet article, j'ai, par souci de clarté, présenté dans un premier mouvement le récit pour pouvoir le confronter à des politiques globales, c'est dans le but d'offrir au lecteur une vision contrastée d'un terrain qui ne lui est probablement pas familier. Mais, arrivé à ce stade de la réflexion, ce souci de clarté se retourne contre moi dans la mesure où le modèle qui engloberait au mieux la dynamique en jeu ne relève pas d'un mouvement transversal, mais d'une boucle de rétroaction. Il me semble évident que la stabilisation du récit et sa sédimentation dépendent des situations vécues et de leur efficacité à donner un contenu phénoménologique à cette histoire racontée. Ni le récit, ni même les nœuds narratifs dégagés par l'analyse ne représentent en aucune manière, la substance stable de la transmission.

Il serait également faux de déclarer que cette manière de lire l'histoire socialiste est une nouvelle fabrication de la génération montante. Supprimez les actes de parole des aînés (en ce compris le récit) ainsi que leurs réalisations matérielles passées et vous supprimez une bonne partie des bases sur lesquelles ce sentiment se construit. Il me semble plutôt que les germes de cette tonalité nostalgique sont lovés là, dans la dynamique des actants humains et matériels qui se nouent et se dénouent en situation.

Un dernier instantané achèvera d'illustrer cette position et le sentiment de la chute qui prédomine aujourd'hui dans les lectures de l'histoire. A côté du centre culturel, sur la plaine herbeuse de la grande place du district, trône un imposant bâtiment à l'état de gros-œuvre. Le rouge des briques tranche sur les dégradés bruns des cabanes de bois qui l'entourent. Un vent hurleur s'engouffre dans les interstices des fenêtres sans vitre et des châssis sans porte. En passant devant ce fantôme qui devait contenir les bureaux administratifs de la coopérative, le médecin du village me lance : «Des années et des centaines de bras pour le construire et même plus suffisamment d'argent ni de motivation pour l'abattre. C'est ça la Mongolie du $21^{\mathrm{e}}$ siècle ! » 


\section{Références bibliographiques}

Badamkhatan, S., 1986. «Les chamanistes du Bouddha vivant ». Traduit du mongol et adapté par MarieDominique Even, Etudes mongoles et sibériennes 17.

Batchuluun, 2004. Khövsgöl aimgiin Rinchinlkhümbe, sümyn tovtch taniltsuulan, manuscrit confié à l'auteur (Présentation succincte du district de Rinchinlkhümbe de la province de Khövsgöl).

Batbayar, Tsendendambyn, 1999. «Stalin’s Strategy in Mongolia, 1932-1936». Mongolian Studies XXII, pp. 1-17.

Bawden, Charles, 1968/1989. The Modern History of Mongolia. London, Kegan Paul.

Berliner, David, 2005. "The Abuses of Memory: Reflections on the Memory Boom in Anthropology ", Anthropological Quarterly 78 (1), pp. 183-197.

BцосH, Maurice, 1995. « Mémoire autobiographique et mémoire historique du passé éloigné », Enquête 2, pp. $59-76$.

Bulag, Uradyn, 1998. Nationalism and Hybridity in Mongolia. Oxford, Clarendon Press.

Denora, Tia, 2000. Music in Everyday life. Cambridge, Cambridge University Press.

DorJnAmjIM, D., 1980. BNMAU-yn soyolyn tüükh. Tergüün bot’ (1921-1940). Ulaanbataar, Ulsyn khevleliin gazar (Histoire culturelle de la République Populaire de Mongolie. Livre premier).

Dundiv, 1912. Manuscrit sans titre transmis à l'auteur.

Halbwachs, Maurice, 1976. Les cadres sociaux de la mémoire. Paris - La Haye, Mouton.

Hennion, Antoine, 1993. La passion musicale. Une sociologie de la médiation. Paris, Métailé.

Hennion, Antoine, Sophie Maisonneuve, et Emilie Gomart, 2000. Figures de l'amateur. Formes, objets, pratiques de l'amour de la musique aujourd'hui. Paris, La documentation Française.

Humphrey, Caroline, 1983. Karl Max Collective: Economy, Society, and Religion in a Siberian Collective Farm. Cambridge, Cambridge University Press.

Humphrey, Caroline et David Sneath, 1999. The End of Nomadism? Society, State, and The Environment in Inner Asia. Durham, Duke University Press.

KundERA, Milan,

1979. Le Livre du rire et de l'oubli. Paris, Gallimard.

2003, « Le théâtre de la mémoire », Le monde diplomatique. Mai, pp. 28-29.

Latour, Bruno, 1994. «Une sociologie sans objet ? Remarques sur l'interobjectivité », Sociologie du travail 4, pp. 587-607.

Legrand, Jacques, 1998. Vents d'herbe et de feutre. Ecrits et dits de Mongolie. Paris, Findakly.

Morawski, Stefan, 1961. «Les péripéties de la théorie du réalisme socialiste. Une petite leçon d’histoire qui n'est pas à dédaigner ». Diogène 36, pp. 120-142.

Pedersen, Morten Axel, 2004. In the Hollow of the Taïga: Landscape, Prominence, and Humour among the Shishged Darxad of Northern Mongolia. Ph.D. dissertation, King's College.

PegG, Carol, 2001. Mongolian Music, Dance, and Oral Narrative. Seattle, University of Washington Press.

Potkanski, Thomas \& SZYNKIEWICZ, Slavoj, 1993. Policy Alternatives for Livestock Development in Mongolia. Brighton, Institute of Development Studies : University of Sussex.

Radley Alan, 1990. «Artefacts, Memory, and a Sense of the Past », in Milddelton D. and D. Edwards (éds.), Collective Remembering. Londres, Sage Publications, pp. 46-60.

Sneath David, 2003. « Lost in the Post: Technologies of Imagination, and the Soviet Legacy in Post-Socialist Mongolia », Inner Asia V (1), pp. 38-52. 


\section{Laurent LEgRAIN}

Wheeler, Alan, 2004. « Moralities of the Mongolian 'Market': A Genealogy of Trade Relations and the Zah Zeel », Inner Asia VI (2), pp. 215-238.

Yondon, 2004. Altan tal negdliin üüsel khögjil. (Edification et développement de la coopérative plaine dorée). Manuscrit confié à l'auteur. 